\title{
Juvenile idiopathic arthritis-associated uveitis: a retrospective analysis from a centre of South Italy
}

\author{
I. Castagna - A. M. Roszkowska 1 - F. Alessandrello • G. W. Oliverio • \\ G. Tumminello $\cdot$ R. Gallizzi $\cdot$ G. Conti $\cdot$ P. Aragona
}

Received: 3 May 2019/ Accepted: 23 September 2019/Published online: 4 October 2019

(C) The Author(s) 2019

\begin{abstract}
Purpose To investigate the clinical and laboratory characteristics of the children affected by juvenile idiopathic arthritis (JIA) who developed uveitis.

Methods In this retrospective study, we have examined data of 109 patients aged from 3 to 16 years, affected by JIA and followed at Paediatrics Rheumatology Clinic and Ophthalmology Clinic of University Hospital of Messina in the period from 2007 to 2017. The main outcome measures were clinical and laboratory findings related to JIA and ocular involvement.
\end{abstract}

I. Castagna · F. Alessandrello · G. W. Oliverio .

G. Tumminello · P. Aragona

Ophthalmology Unit, Department of Biomedical

Sciences, University Hospital of Messina, Messina, Italy

A. M. Roszkowska

Ophthalmology Section, Faculty of Medicine and Health Sciences, Andrzej Frycz Modrzewski Kraków University, Kraków, Poland

R. Gallizzi

Unit of Pediatrics, Department of Human Pathology in Adulthood and Childhood "G. Barresi", University of Messina, Messina, Italy

G. Conti

Pediatric Nephrology and Rheumatology Unit, University Hospital of Messina, Messina, Italy

A. M. Roszkowska ( $\bowtie)$

A.O.U. Policlinico G. Martino Messina, Via Consolare

Valeria 1, 98125 Messina, Italy

e-mail: aroszkowska@unime.it
The prevalence of ocular signs and symptoms was determined and correlated with age.

Results Twenty-one (19.3\%) subjects developed uveitis. Two different peaks of age with ocular involvement were registered. The first occurred between 4 and 6 years and the second between 10 and 12 years. All subjects in the first group resulted to be female, presented oligoarticular arthritis and chronic anterior uveitis. In the second group, the $84 \%$ of patients were male with different types of JIA and acute anterior uveitis. The prevalence of ocular complications was higher in the first group.

Conclusions Two peaks of age emerged and were characterized by different clinical outcomes of arthritis and ocular involvement. The first occurred between 4 and 6 years and interested females affected by oligoarticular JIA who develop chronic anterior uveitis. The second appeared at 10-12 years and interested older males affected by different types of JIA with acute anterior uveitis. Early diagnosis and cooperation between paediatric rheumatologist and ophthalmologist are of great importance in the proper management of JIA children with uveitis.

Keywords Juvenile idiopathic arthritis - Uveitis · Age $\cdot$ Epidemiology $\cdot$ Risk factors $\cdot$ Children

\section{Introduction}

Juvenile idiopathic arthritis (JIA) represents the most common chronic rheumatic disease of childhood, 
determining short- and long-term disability. JIA is related to heterogeneous group of articular inflammations with unknown aetiology, with onset before the age of 16 years and duration of at least 6 weeks [1].

The International League of Associations for Rheumatology (ILAR) classified JIA into seven subgroups: systemic JIA, oligoarticular JIA, rheumatoid factor (RF)-positive polyarticular JIA, RF-negative polyarticular JIA, enthesitis-related arthritis (ERA), psoriatic arthritis (PsA) and undifferentiated JIA [2] which are summarized in Table 1.

The oligoarticular arthritis represents the most frequent form $(27-56 \%)$ [1]. It is characterized by inflammation of four or fewer joints, early onset throughout childhood, with a peak age of onset at around 2 years. Females are interested five times more as males. This group of arthritis shows a strong positivity of antinuclear antibodies (ANA) with uveitis occurring in around $20 \%$ of cases.

Uveitis is the most common and severe extraarticular manifestation of JIA [3, 4]. It is reported in up to $25 \%$ of patients with JIA, with a greater prevalence in Europe [5, 6]. The course of uveitis is independent of arthritis activity, and despite therapy, a great number of children still develop ocular complications, associated with high prevalence of visual loss [1].

Usually the patients develop uveitis within 3 years after diagnosis of JIA, but in less than $10 \%$ of subjects uveal inflammation precedes articular inflammation $[1,3]$. Uveitis can be classified according to the Standardisation of Uveitis Nomenclature (SUN) International Working Group. It is described as anterior, intermediate, posterior and panuveitis in relation to anatomical localization of ocular inflammation. Uveitis onset can be sudden or insidious, its duration limited in time or persistent and its course acute, recurrent or chronic [7].

JIA-associated uveitis commonly manifest as chronic anterior uveitis, which is often clinically silent, but can lead to serious sight-threatening complications. This form of uveitis is typically associated with oligoarticular and RF-negative polyarticular arthritis with particular risk for girls who develop JIA within 3 years of age with ANA positivity [1, 3, 8-10]. In contrast, boys with ERA HLA-B27 positivity develop most frequently acute anterior uveitis, which is symptomatic, unilateral and episodic $[3,8,10,11]$.

Treatment includes topical therapies with cycloplegics, steroids and NSAID and systemic immunosuppressive therapies based on steroids, synthetic DMARDs such as methotrexate or biologic drugs such as adalimumab and infliximab $[8,12]$.

Early diagnosis and treatment of uveitis is essential to prevent ocular complications, which can develop in up to $37 \%$ of cases, leading to visual loss [6]. Structural complications result from both the disease itself and its treatments and comprise band keratopathy, posterior synechiae, cataract, glaucoma, macular cystoid oedema, hypotony and epiretinal membrane formation $[6,8,10,12-20]$.

The purpose of this study is to analyse the clinical and laboratory characteristics of children affected by juvenile idiopathic arthritis who developed uveitis and to correlate the prevalence of ocular signs and symptoms with age.

Table 1 International League of Associations for Rheumatology (ILAR) JIA classification [1, 25]

\begin{tabular}{|c|c|c|c|c|c|}
\hline & $\begin{array}{l}\text { Frequency } \\
(\%)\end{array}$ & Onset age & Sex ratio & Laboratory & $\begin{array}{l}\text { Uveitis } \\
\text { risk }\end{array}$ \\
\hline Oligoarticular & $27-56$ & Early childhood; peak at $2-4$ years & $F / M=5: 1$ & $\mathrm{ANA}+75 \%$ & $20-30 \%$ \\
\hline $\begin{array}{l}\text { Polyarticular RF } \\
\text { negative }\end{array}$ & $11-28$ & $\begin{array}{l}\text { Biphasic distribution; early peak at } 2-4 \text { years and later } \\
\text { peak at } 6-12 \text { years }\end{array}$ & $F / M=3: 1$ & $\mathrm{ANA}+40 \%$ & $10 \%$ \\
\hline $\begin{array}{l}\text { Polyarticular RF } \\
\text { positive }\end{array}$ & $2-7$ & Late childhood or adolescence & $F / M=3: 1$ & $\mathrm{RF}+$ & Rare \\
\hline Systemic & $4-17$ & Throughout childhood & $F / M=1: 1$ & ANA- & Rare \\
\hline Enthesitis related & $3-11$ & Late childhood or adolescence & $F / M=1: 3$ & HLA-B27 & $10-15 \%$ \\
\hline Psoriatic & $2-11$ & $\begin{array}{l}\text { Biphasic distribution; early peak at } 2-4 \text { years and later } \\
\text { peak at } 9-11 \text { years }\end{array}$ & $F / M=2: 1$ & & $10-20 \%$ \\
\hline Other & $11-21$ & - & - & - & - \\
\hline
\end{tabular}




\section{Patients and methods}

In this retrospective study, we analysed data of patients affected by JIA and followed in Paediatrics Rheumatology and Ophthalmology Clinics of University Hospital of Messina between January 2007 and December 2017.

The study included 109 Italian children fulfilling the criteria for JIA such as age between 3 and 16 years, arthritis during at least 6 weeks and not other detectable cause of arthritis. Table 2 represents parameters considered for evaluation.

JIA was diagnosed according to the ILAR classification, and the SUN classification was used to diagnose and grade the associated uveitis and its activity $[2,7]$.

We defined as acute uveitis a sudden onset episode with limited duration, recurrent uveitis repeated episodes separated by periods of 3 months inactivity without treatment and chronic as persistent uveitis with relapse in 3 months after discontinuing treatment [7]. The ILAR categories, uveitis localization, time between JIA diagnosis and uveitis onset, and age were determined for each subject. Visual acuity (VA) was evaluated using decimal chart, and it was considered reduced if less than 0.8 .

Statistical analysis was performed using the SPSS 11.0 for Windows package. The numerical data were expressed as means with standard deviation and range relatively to whole analysed population. To analyse the subgroup with uveitis, the medians with associated 25th and 75th percentiles were used, together with the categorical variables as numbers and percentage. The Chi-square test for categorical data and Student's $t$ test were used for statistical analysis when appropriate and differences were considered significant if $p$ value was less than 0.05 .

The study obtained approval of the Ethical Committee of University Hospital of Messina.

\section{Results}

Twenty-one (19.3\%) patients (28 eyes) affected by JIA developed uveitis during the follow-up. Fifteen were female $(71.4 \%)$ and six were male $(28.6 \%)$ with a female-to-male ratio of $2.5: 1$. The main characteristics of children who developed uveitis are listed in Tables 3 and 4 .
Table 2 Patients' data considered for evaluation

Table 3 Characteristics of children who developed uveitis

\begin{tabular}{lll}
\hline Epidemiologic data & Laboratory data & Clinical data \\
\hline Gender & ANA & Symptoms \\
Age of JIA onset & HLA-B27 & Visual acuity \\
Number of affected joints & RF & Anterior segment signs \\
Age of uveitis onset & & Posterior segment signs \\
Time between JIA diagnosis and uveitis onset & & Uveitis treatment \\
\hline
\end{tabular}

Patients with JIA-associated uveitis

Patients, $n(\%)$

$21 / 109(19.3 \%)$

Female $n(\%)$, male $n(\%)$

15 (71.4\%), 6 (28.6\%)

JIA onset, mean age

5 years $(61 \pm 38$ months $)$

Time between JIA onset and uveitis onset

3-5 years before JIA

$2(10 \%)$

Concurrently with JIA

$2(10 \%)$

2.5 years after JIA (34 \pm 50 months)

$17(80 \%)$

Uveitis type

Anterior monolateral uveitis

$14(66 \%)$

Anterior bilateral uveitis

$5(24 \%)$

Bilateral intermediate uveitis
$2(10 \%)$ 
Table 4 ILAR categories in children who developed uveitis

\begin{tabular}{llll}
\hline ILAR categories & ANA + & ANA - & $p$ value* \\
\hline Oligoarticular JIA & 13 & 1 & $\mathbf{0 . 0 0 0 9}$ \\
Polyarticular JIA & 3 & 2 & 0.5 \\
ERA & 1 & 0 & 0.5 \\
Psoriatic arthritis & 0 & 1 & 0.5 \\
Total & 17 & 4 & $\mathbf{0 . 0 0 3 5}$ \\
\hline
\end{tabular}

Bold values indicate the statistically significant

*Fisher's test

Mean age at diagnosis of JIA was about 5 years (61 \pm 38 months). Mean time between JIA diagnosis and uveitis onset was about 2.5 years (34 \pm 50 months) for 17 patients $(80 \%)$, but in two cases uveitis preceded arthritis of 3 and 5 years. The oligoarticular form of JIA affected $66 \%$ of patients, in $80 \%$ of patients ANA were found and in $90 \%$ of patients anterior uveitis occurred.

Age of patients at uveitis manifestation was determined and is represented in Fig. 1. The median age of patients with uveitis was 8 (IQR 5-12). There is an immediate evidence of two main peaks of age. The first occurred between 4 and 6 years (first quartile) and the second between 10 and 12 years (third quartile) with only sporadic manifestation in other age. These two groups of subjects in relation to age peak were considered for statistical analysis. There was a statistically significant difference between the ages of the two subgroups of patients $(p<0.05)$.

Children included in the first group that aged from 4 to 6 years, resulted to be all female, they presented the ILAR category of oligoarticular arthritis and developed chronic manifestations of anterior uveitis. In the group of patients aged from 10 to 12 years, $84 \%$ were male, affected by other types of JIA $(50 \%$ oligoarticular JIA, $16.3 \%$ polyarticular JIA, 16.3\% psoriatic arthritis, $16.3 \%$ ERA HLA-B27+), and they developed acute manifestations of anterior uveitis (Table 5).

In the first group, a chronic anterior uveitis usually occurred as so-called white uveitis, characterized by apparent lack of evident signs and symptoms, but with several signs observed on the slit lamp examination. All eyes had keratic precipitates, Tyndall and miosis, and during the follow-up, they developed several complications.

In the second group, all patients were symptomatic with pain and photophobia and showed a clear clinical picture of acute anterior uveitis, characterized by "red eye" due to the ciliary injection, keratic precipitates, Tyndall, miosis, aqueous flare and in two eyes hypopyon.

Six eyes (28\%) had reduced visual acuity. As comparing visual acuity of the two groups, nonstatistically significant difference was found $(p=0.54)$, suggesting that visual prognosis is the same for both type of uveitis, acute and chronic, despite different clinical presentations and complications (Tables 6, 7).

The therapeutic management of uveitis therapy was conducted according to the treatment algorithm [21]. Worsening activity was defined in a two-step increase in level of inflammation (e.g. anterior chamber cells, vitreous haze) or an increase from grade 3 to 4; all patients with inactive disease for 3 months after
Fig. 1 Age distribution of the patients with uveitis with number of affected eyes

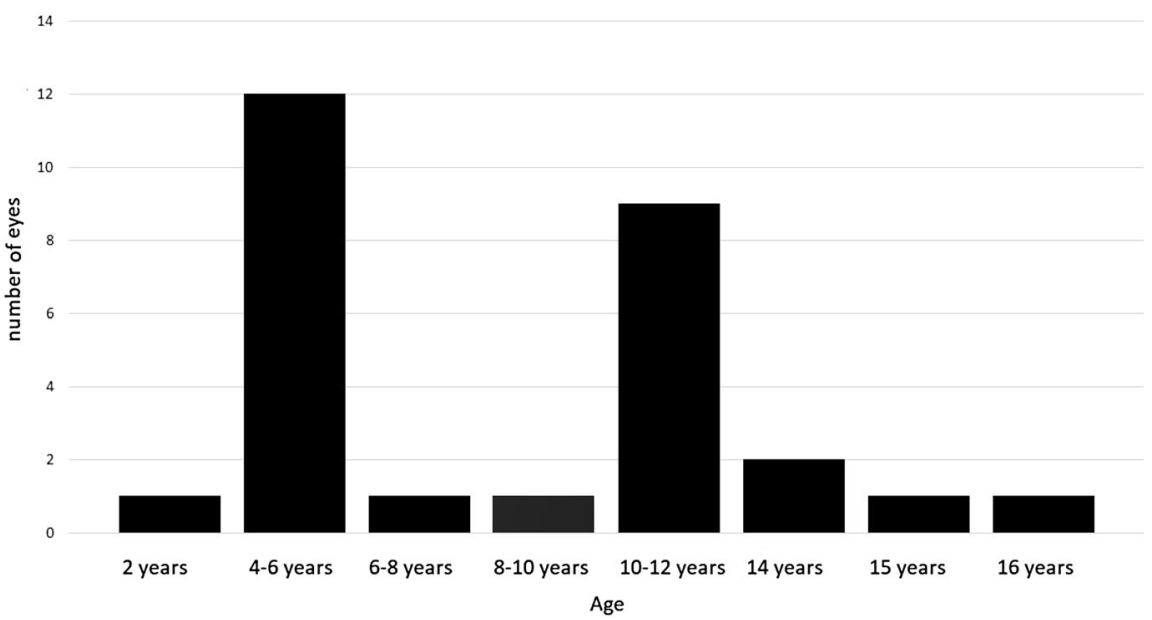


Table 5 Characteristics of the two subgroups in relation to the peak of age
Bold values indicate the statistically significant

*Fisher's test

Table 6 Clinical signs and symptoms in the two subgroups statistically significant

*Fisher's test

Table 7 Complications in the two subgroups

Bold values indicate the statistically significant

*Fisher's test
Bold values indicate the

\begin{tabular}{llll}
\hline Age & 4-6 years & 10-12 years & $p$ value* \\
\hline Patients, $n$ & 9 & 6 & 0.3 \\
Eyes, $n$ & 12 & 9 & 0.33 \\
Sex & 9 Females (100\%) & 5 Males (84\%), 1 female (16\%) & $\mathbf{0 . 0 0 2}$ \\
ILAR & Oligoarticular JIA 100\% & Oligoarticular JIA 50\% & $\mathbf{0 . 0 4}$ \\
& & Others 50\%: & \\
& & (polyarticular JIA 16.3\%) & \\
& & (psoriatic arthritis 16.3\%) & \\
Uveitis type & Anterior uveitis & (ERA HLA-B27+ 16.3\%) & \\
\hline
\end{tabular}

\begin{tabular}{lllc}
\hline Clinical signs and symptoms & Age 4-6 (eyes $n=12)$ & Age 10-12 (eyes $n=9)$ & $p$ value* \\
\hline Pain & 0 & $9(100 \%)$ & $<\mathbf{0 . 0 0 0 0 1}$ \\
Photophobia & 0 & $9(100 \%)$ & $<\mathbf{0 . 0 0 0 0 1}$ \\
Perichoretic injections & 0 & $9(100 \%)$ & $<\mathbf{0 . 0 0 0 0 1}$ \\
Keratic precipitates & $12(100 \%)$ & $9(100 \%)$ & 1 \\
Tyndall & $12(100 \%)$ & $9(100 \%)$ & 1 \\
Flare & $12(100 \%)$ & $9(100 \%)$ & 1 \\
Ipopion & 0 & $2(22 \%)$ & 0.17 \\
Koeppe nodules & $2(16 \%)$ & 0 & 0.49 \\
Miosis & $12(100 \%)$ & $9(100 \%)$ & 1 \\
\hline
\end{tabular}

\begin{tabular}{llll}
\hline Complications & Age 4-6 (eyes $n=12)$ & Age 10-12 (eyes $n=9)$ & $p$ value* \\
\hline Band keratopathy & $4(33 \%)$ & 0 & 0.104 \\
Anterior synechiae & 0 & 0 & 1 \\
Posterior synechiae & $8(66 \%)$ & $2(22 \%)$ & 0.08 \\
Cataract & $4(33 \%)$ & 0 & 0.103 \\
Hypotony & $10(83 \%)$ & 0 & $\mathbf{0 . 0 0 0 2}$ \\
Increased IOP & 0 & 0 & 1 \\
Cystoid macular oedema & $4(33 \%)$ & $2(22 \%)$ & 0.66 \\
Reduced visual acuity & $4(33 \%)$ & $2(22 \%)$ & 0.66 \\
Phthisis & 0 & 0 & 1 \\
\hline
\end{tabular}

discontinuing all treatments were in clinical remission [7]. All 21 patients were initially treated with topical patients achieved permanent clinical remission until today (Fig. 2).

\section{Discussion}

In our cohort of young patients with JIA, 19.3\% developed uveitis. The oligoarthritis was the most common form of JIA (66\%), $71 \%$ were female and the majority of children had ANA positivity (80\%). 
Fig. 2 Flow chart therapy

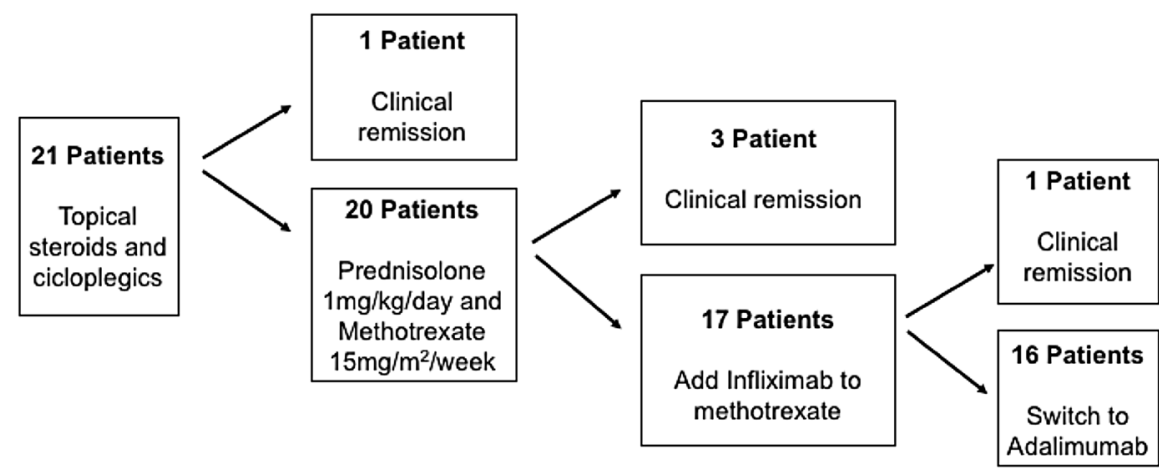

According to the literature, the prevalence of JIAassociated uveitis ranged from 11.6 to $30 \%$ [24, 25]. Papadopoulou et al. [26] conducted a retrospective study on a cohort of 299 Swedish children with JIA and found that $11 \%$ of them developed uveitis, prevalent subtype of JIA was oligoarticular (75\%), $78 \%$ of patients were girls and almost all children were ANA positive (97\%) [26]. Angeles-Han et al. [24] found similar results, confirming uveitis risk markers such as younger age at arthritis onset, oligoarticular JIA and ANA positivity. As to the onset of uveal inflammation, in the our study $80 \%$ of children developed uveitis 2.5 years after JIA diagnosis, and only in two cases (10\%) it was already present before arthritis. In a recent prospective longitudinal study Noradal et al. [27], uveitis developed at average interval of 9.6 months after arthritis onset (range 4.7-9.4 years). Heiligenhaus et al. [28] found uveitis preceding arthritis with a prevalence of $3-7 \%$.

In the our study, two peaks of age with uveitis onset were clearly evidenced with females developing uveitis earlier, between 4 and 6 years, as compared to males with age of onset comprised between 10 and 12 years.

Additionally, the present study confirmed that different subtypes of JIA characterize both groups together to the different types of uveitis. In fact, younger girls were affected by oligoarticular JIA developing chronic anterior uveitis, whereas the older boys, who had other subtypes of JIA, experienced acute anterior uveitis.

In JIA patients, the ocular inflammation is most frequently diagnosed between the fourth and the sixth years of life $[5,14,15]$. In fact, the literature reports a number of risk factors for JIA-associated chronic anterior uveitis such as female gender, younger age, oligoarticular JIA and ANA positivity. In contrast, male gender, ERA and HLA-B27+ predispose to acute anterior uveitis [6]. There is not common agreement on gender and JIA subtype as risk factors for chronic anterior uveitis. Indeed, Saurenmann et al. [4] suggested that the risk of JIA-associated uveitis is strongly related to the patient's age at the time of arthritis onset and ANA positivity in girls but not in boys, and it is independent of JIA subtypes. Calandra et al. [29] proposed that risk is related to younger age at onset of JIA and to ANA positivity, but not to gender instead. Acute anterior uveitis is the extraarticular complication of different types of JIA, such as enthesitis-related arthritis, spondylitis and psoriatic arthritis, which are frequently characterized by HLAB27 positivity (ERA and spondylitis), and develop in older children [5, 6, 11, 30]. Furthermore, when psoriatic arthritis develop later in childhood, symptomatic recurrent acute anterior uveitis is typical, rather than chronic uveitis, which develop in early childhood [31]. Saurenmann et al. [32] found that males were more likely to have symptomatic uveitis, an older age at diagnosis of uveitis and ERA and psoriatic JIA compared to females. Our results are in line with those of other groups, but, to our knowledge, this is the first study that clearly identified two different peaks of age related to chronic and acute uveitis and type of JIA.

The our study confirms that young patients with chronic anterior uveitis experienced more structural complications with respect to the patients with acute anterior uveitis as previously reported [3, 5, 8]. Chronic anterior uveitis is clinically silent and frequently very young children are unable to refer their symptoms, and that can lead to a late diagnosis. Differently, the acute anterior uveitis causes red eye, pain and photophobia leading to an early 
ophthalmological referral and treatment. For this reason, all patients at risk of JIA-associated uveitis should be screened according to guidelines available in several countries [15, 33-35].

In our population, $28 \%$ eyes had reduced visual acuity up to 0.4 . According to the literature, the risk of reduced visual acuity to 0.4 ranges from 13 to $26 \%$ and of reduction to 0.1 ranges from 5 to 9\%.[11, 17, 19, 36-39].

Comparing visual acuity of two groups, nonstatistically significant difference was found, suggesting that visual prognosis is the same for both types of uveitis, acute and chronic, although clinical presentations and complications are very different. This is due to the efficiency of screening plans, which leads to early diagnosis and to the efficacy of treatment.

It could be concluded that uveitis is the most severe extra-articular complication of JIA, with a significant number of children still developing sight-threatening complications, associated with high prevalence of visual loss, despite therapy. There are two peaks of age related to different clinical characteristics of arthritis and ocular involvement: younger female aged from 4 to 6 years, are affected by oligoarticular JIA and develop chronic anterior uveitis; older male aged from 10 to 12 years, are affected by different types of JIA and develop acute anterior uveitis. A close cooperation between paediatric rheumatologist and ophthalmologist is advisable for early diagnosis, following screening plans to be established according to risk factors for each patient, particularly focusing on age and clinical characteristics.

Authors' contribution All authors contributed to the acquisition of data, writing and revision of this manuscript.

\section{Compliance with ethical standards}

Conflict of interest The authors have no conflict of interest related to this study.

Ethical approval This study was approved by the Ethical Committee of the University Hospital of Messina Prot.: 371; $14 / 11 / 2018$.

Open Access This article is distributed under the terms of the Creative Commons Attribution 4.0 International License (http:// creativecommons.org/licenses/by/4.0/), which permits unrestricted use, distribution, and reproduction in any medium, provided you give appropriate credit to the original author(s) and the source, provide a link to the Creative Commons license, and indicate if changes were made.

\section{References}

1. Ravelli A, Martini A (2007) Juvenile idiopathic arthritis. Lancet 369:767-778

2. Petty RE et al (2004) International League of Associations for Rheumatology classification of juvenile idiopathic arthritis: second revision, Edmonton, 2001. J Rheumatol 31(2):390-392

3. Clarke SLN, Sen ES, Ramanan AV (2016) Juvenile idiopathic arthritis-associated uveitis Clarke et al. Pediatric Rheumatol 14 (27):1-11. https://doi.org/10.1186/s12969016-0088-2

4. Saurenmann RK, Levin AV, Feldman BM, Laxer RM, Schneider R, Silverman ED (2010) Risk factors for development of uveitis differ between girls and boys with juvenile idiopathic arthritis. Arthritis Rheum 62(6):1824-1828

5. Heiligenhaus A, Minden K, Föll D, Pleyer U (2015) Uveitis in juvenile idiopathic arthritis. Dtsch Arztebl Int 112:92-100

6. Angeles-Han ST, Yeh S, Vogler LB (2013) Updates on the risk markers and outcomes of severe juvenile idiopathic arthritis-associated uveitis. Int J Clin Rheumtol. https://doi. org/10.2217/ijr.12.83

7. Jabs DA, Nussenblatt RB, Rosenbaum JT (2005) Standardization of uveitis nomenclature (SUN) working group. Standardization of uveitis nomenclature for reporting clinical data. Results of the first international workshop. Am J Ophthalmol 140(3):509e16

8. Sen ES, Ramanan AV (2017) Juvenile idiopathic arthritisassociated uveitis, best practice \& research. Clin Rheumatol 31(4):517-534

9. Zygmunt A, Lipińska J, Biernacka-Zielińska M et al (2018) Comparison of uveitis in the course of juvenile idiopathic arthritis with isolated uveitis in children-own experiences. Reumatologia 56(3):149-154

10. Abu Samra K, Maghsoudlou A, Roohipoor R, ValdesNavarro M, Lee S, Stephen Foster C (2016) Current treatment modalities of JIA-associated uveitis and its complications: literature review. Ocul Immunol Inflamm. https:// doi.org/10.3109/09273948.2015.1115878

11. Woreta F, Thorne JE, Jabs DA, Kedhar SR, Dunn JP (2007) Risk factors for ocular complications and poor visual acuity at presentation among patients with uveitis associated with juvenile idiopathic arthritis. Am J Ophthalmol 143:647-655

12. Gicchino MF, Calandra S, Muratore V, Giancane G, Filocamo G, Ravelli A (2017) Update sulla terapia dell'uveite associata all'artrite idiopatica giovanile con agenti biologici. Reumatologia pediatrica luglio-settembre 47(187):205-212

13. Heiligenhaus A et al (2012) Proposed outcome measures for prospective clinical trials in juvenile idiopathic arthritisassociated uveitis: a consensus effort from the multinational interdisciplinary working group for uveitis in childhood. Arthritis Care Res (Hoboken) 64(9):1365-1372

14. Carvounis PE, Herman DC, Cha S, Burke JP (2006) Incidence and outcomes of uveitis in juvenile rheumatoid arthritis: a synthesis of the literature. Graefes Arch Clin Exp Ophthalmol 244:281-290

15. Heiligenhaus A, Niewerth M, Ganser G, Heinz C, Minden K, German Uveitis in Childhood Study Group (2007) 
Prevalence and complications of uveitis in juvenile idiopathic arthritis in a population-based nation-wide study in Germany: suggested modification of the current screening guidelines. Rheumatology (Oxford) 46:1015-1017

16. Thorne JE, Woreta F, Kedhar SR, Dunn JP, Jabs DA (2007) Juvenile idiopathic arthritis-associated uveitis: incidence of ocular complications and visual acuity loss. Am J Ophthalmol 143:840-846

17. Edelsten C, Lee V, Bentley CR, Kanski JJ, Graham EM (2002) An evaluation of baseline risk factors predicting severity in juvenile idiopathic arthritis associated uveitis and other chronic anterior uveitis in early childhood. Br J Ophthalmol 86:51-56

18. De Boer J, Wulffraat N, Rothova A (2003) Visual loss in uveitis of childhood. Br J Ophthalmol 87:879-884

19. Kump LI, Cervantes-Castaneda RA, Androudi SN, Foster CS (2005) Analysis of pediatric uveitis cases at a tertiary referral center. Ophthalmology 112:1287-1292

20. Sijssens KM, Rothova A, Berendschot TT, de Boer JH (2006) Ocular hypertension and secondary glaucoma in children with uveitis. Ophthalmology 113:853-859

21. Bou R, Adan A, Borras F, Bravo B, Calvo I, De Inocencio J, et al (2015) Clinical management algorithm of uveitis associated with juvenile idiopathic arthritis: interdisciplinary panel consensus. Rheumatol Int 35(5):777e85

22. Cecchin V et al (2018) Longterm safety and efficacy of adalimumab and infliximab for uveitis associated with juvenile idiopathic arthritis. J Rheumatol 45(8):1167-1172

23. Schmeling $\mathrm{H}$ et al (2014) Efficacy and safety of adalimumab as the first and second biologic agent in juvenile idiopathic arthritis. Arthritis Rheumatol 66(9):2580-2589

24. Angeles-Han ST, Pelajo CF, Vogler LB, Rouster-Stevens K, Kennedy C, Ponder L, et al (2013) Risk markers of juvenile idiopathic arthritis-associated uveitis in the childhood arthritis and rheumatology research alliance (CARRA) registry. J Rheumatol 40(12):2088e96

25. Moradi A, Amin RM, Thorne JE (2014) The role of gender in juvenile idiopathic arthritis-associated uveitis. J Ophthalmol 2014:461078

26. Papadopoulou M, Zetterberg M, Oskarsdottir S et al (2017) Assessment of the outcome of ophthalmological screening for uveitis in a cohort of Swedish children with juvenile idiopathic arthritis. Acta Ophthalmol 95(7):741-747. https://doi.org/10.1111/aos.13388

27. Nordal E, Rypdal V, Christoffersen T, Aalto K, Berntson L, Fasth A et al (2017) Incidence and predictors of Uveitis in juvenile idiopathic arthritis in a Nordic long-term cohort study. Pediatr Rheumatol Online J 15(1):66
28. Heiligenhaus A, Heinz C, Edelsten C, Kotaniemi K, Minden K (2013) Review for disease of the year: epidemiology of juvenile idiopathic arthritis and its associated uveitis: the probable risk factors. Ocul Immunol Inflamm 21(3):180e91

29. Calandra S et al (2014) Female sex and oligoarthritis category are not risk factors for uveitis in Italian children with juvenile idiopathic arthritis. J Rheumatol 41(7):1416-1425

30. Prakken B, Albani S, Martini A (2011) Juvenile idiopathic arthritis. Lancet 377:2138-2149

31. Kesen MR, Setlur V, Goldstein DA (2008) Juvenile idiopathic arthritis-related uveitis. Int Ophthalmol Clin 48(3):21-38

32. Saurenmann RK, Levin AV, Feldman BM, Rose JB, Laxer RM, Schneider R, Silverman ED (2007) Prevalence, risk factors, and outcome of uveitis in juvenile idiopathic arthritis: a long-term followup study. Arthritis Rheum 56(2):647-657

33. British Society for Paediatric and Adolescent Rheumatology (2006) Guidelines for screening for uveitis in juvenile idiopathic arthritis. Royal College of Ophthalmology, London

34. National Guideline Clearinghouse (2012) Best evidence statement (BESt). Screening for uveitis in children with juvenile idiopathic arthritis (JIA)

35. Cassidy J, Kivlin J, Lindsley C, Nocton J (2006) Section on rheumatology, section on ophthalmology. Ophthalmologic examinations in children with juvenile rheumatoid arthritis. Pediatrics 117(5):1843e5

36. Edelsten C, Reddy MA, Stanford MR, Graham EM (2003) Visual loss associated with pediatric uveitis in English primary and referral centers. Am J Ophthalmol 135:676-680

37. Saurenmann RK, Levin AV, Feldman BM et al (2007) Prevalence, risk factors, and outcome of uveitis in juvenile idiopathic arthritis: a long-term followup study. Arthritis Rheum 56:647-657

38. Sabri K, Saurenmann RK, Silverman ED, Levin AV (2008) Course, complications, and outcome of juvenile arthritisrelated uveitis. J AAPOS 12:539-545

39. Smith JA, Mackensen F, Sen HN et al (2009) Epidemiology and course of disease in childhood uveitis. Ophthalmology 116:1544-1551

Publisher's Note Springer Nature remains neutral with regard to jurisdictional claims in published maps and institutional affiliations. 